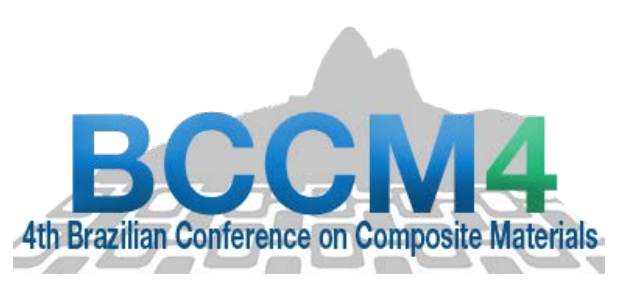

\title{
COMPOSITE STRUCTURAL BEHAVIOUR PREDICTION WITH THE SURROGATE MODEL AND TRACE-BASED APPROACH
}

\author{
Eduardo Gerhardt ${ }^{(1)}$, Rogério J. Marczak ${ }^{(2)}$ and Sandro C. Amico ${ }^{(3)}$ \\ (1) SENAI Institute for Innovation in Polymers Engineering, Brazil. \\ (2) Department of Mechanical Engineering, Federal University of Rio Grande do Sul, Brazil. \\ (3) Department of Materials Engineering, Federal University of Rio Grande do Sul, Brazil.
}

https://doi.org/10.21452/bccm4.2018.16.11

\begin{abstract}
An approach for the prediction of a composite tube structural behavior is presented. An estimate of the elastic properties of the material is accessed by the development of a knowledge-based surrogate model and the use of a trace-design approach, which is compared with experimental tests. The estimate is applied to a finite element model simulation for buckling analysis using both linear and non-linear methods. Buckling simulation results are then compared with the result of an experimental hydrostatic pressure test. It is demonstrated that this methodology can be a valuable tool in the early stages of a structural design.
\end{abstract}

\section{INTRODUCTION}

Filament winding process produces high quality composite polymer tubes and vessels. Because of its peculiar features, this technique is most suitable to produce surfaces of revolution [1]. However, most standardize procedures for characterizing composite materials demand flat coupons. Flat coupons when made by filament winding will not represent the real state of that material in a revolution shape due to differences in compaction influencing the void and fiber content, for example [1]. Some standard methods for tubular specimen characterization are available, although requiring laborious and time-consuming tasks.

Pursuing the reduction in effort required for composite characterization, Tsai and co-workers have proposed the trace-based approach [2]. They proposed the use of a master-ply for carbonepoxy composites that corresponds to a statistical relation with the stiffness parameters of that material system. With the master-ply, the elastic properties of a ply can be estimated after measuring the elastic modulus in the fiber direction only [3]. Recently, it was proposed that a structure designed by the trace approach could have its material properties scaled by coupons taken from that structure for predicting its behavior [4]. 
Buckling of composite cylinders is a critical issue since it represents the loss of structural stability leading to premature collapse. Buckling predictions of composite structures is affected by several factors, among them geometrical imperfections, actual mechanical properties of the material, over-simplified numerical model assumptions, and sensitivity to internal defects [5].

This study focuses on composite tube structural behavior prediction. The material elastic properties are assessed by a surrogate model and using the trace-based approach, which are applied to radial ring compression test. Finite element model simulation for buckling analysis using both linear and non-linear methods is performed and compared with experimental hydrostatic pressure test results.

\section{METHODS}

\subsection{Trace-based approach}

From the linear stress-strain relation of a unidirectional ply, the trace is defined as an invariant equal to the sum of diagonal elements of the stiffness matrix [Q]. The trace value is obtained in the form of Equation (1) in the principal directions of the ply [6].

$$
\operatorname{Tr}(Q)=Q_{11}+Q_{22}+2 Q_{66}=Q_{x x}+Q_{y y}+2 Q_{s s}
$$

With the trace value it is possible to obtain the master-ply which is given by the tracenormalized stiffness parameters $\left[Q_{i j}{ }^{*}\right]$ and the trace-normalized modulus $\left[E_{X}{ }^{*}\right]$ using Equations (2) and (3), respectively.

$$
\begin{aligned}
& {\left[Q_{i j}{ }^{*}\right]=\frac{\left[Q_{i j}\right]}{\operatorname{Tr}(Q)}} \\
& {\left[E_{x}^{*}\right]=\frac{E_{x}}{\operatorname{Tr}(Q)}}
\end{aligned}
$$

The flexural laminate stiffness trace $\operatorname{Tr}\left(D^{*}\right)$ can also be obtained and has the same value of $\operatorname{Tr}(Q)$. Thus, the flexural stiffness parameter $D_{22}{ }^{*}$ can be calculated by Equation (4) and the tracenormalized flexural stiffness parameter $D_{22}{ }^{* *}$ by Equation (5).

$$
\begin{aligned}
& {\left[D_{22}{ }^{*}\right]=\frac{12\left[D_{22}\right]}{t^{3}}} \\
& {\left[D_{22}^{* *}\right]=\frac{D_{22}{ }^{*}}{\operatorname{Tr}(Q)}}
\end{aligned}
$$

\subsection{Material properties scaling}

Two composite tubes were manufactured by filament winding with a carbon/epoxy towpreg (Toray T700-12K-50C / UF3369 epoxy resin) using a MF Tech robotic system. The stacking sequence adopted was [90/-90/60/-60/90/-90 $]_{\mathrm{T}}$. From these cylinders, a ring section was cut and subjected to radial compression between parallel plates on a universal testing machine, at 5 $\mathrm{mm} / \mathrm{min}$ and $23{ }^{\circ} \mathrm{C}$, to obtain force-displacement curves (Figure 1a). One test was run in a monotonic way until failure. Another test was run in a cyclic way, loading and unloading the sample at ever-higher loads in an attempt to monitor damage evolution in the specimen. These tests were necessary to validate the linear elastic behaviour of the material. 
The unidirectional ply elastic properties of this material were also characterized [7] from flat coupons produced by filament winding. As already commented, these material properties may differ from those obtained when the towpreg is wound over a cylindrical mandrel. To overcome this, material properties scaling procedure was developed, as follows. A monotonic test was simulated with the finite element method applying the original elastic properties (Figure 1b). The simulation was modelled in ABAQUS software [8] using quadratic shell elements, large displacement field hypothesis, ring section dimensions (thickness, radius and width) equal to the actual test specimen, and the mesh size was defined after a mesh sensitivity analysis study.
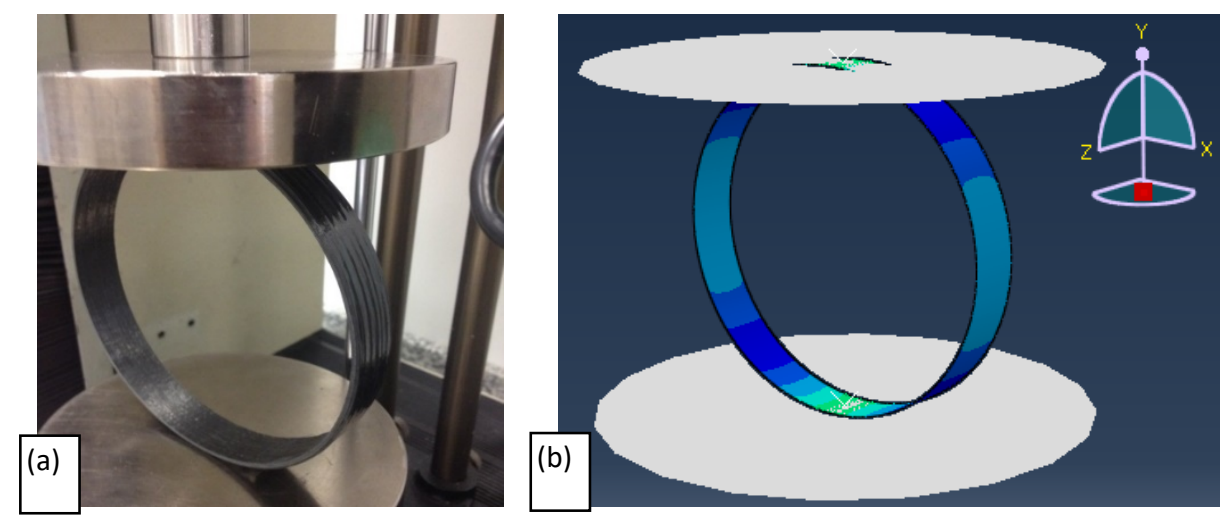

Figure 1 - Preliminary experimental (a) and simulated (b) ring compression test.

With the experimental and numerical curves, a knowledge-based surrogate model was developed based on the analytical solution found in reference [9] and shown as Equation (6). This analytical solution refers to the displacement of a ring compressed by a point load and does not take into account the contact between the compression plates and the ring or the large displacements hypothesis.

$$
w=\frac{P R^{3}}{4 E I}\left(\frac{\pi}{2}-\frac{4}{\pi}\right)
$$

where $w$ is half the displacement on the compressed ring, $P$ is the applied force, $E$ is the elastic modulus of an isotropic material and $I$ is the section moment of inertia.

From the moment-curvature equations [9], it is possible to combine the laminate bendingstiffness parameter $D_{22}$, defined in Equation (7), with Equation (6) to obtain the forcedisplacement relation as a function of $D_{22}$, as shown in Equation (8).

$$
\begin{aligned}
& D_{22} \approx \frac{E I}{b} \\
& P=13.4428 \frac{b w}{R^{3}} D_{22}
\end{aligned}
$$

where $D_{22}$ is the bending parameter of the ABD matrix, $b$ is the width of the ring section.

From Equation (8), the knowledge-based surrogate model, Equation (9), was obtained implementing an unknown function parameter determined through a numerical procedure for error minimization between the experimental and the simulated force-displacement curves. 


$$
P=13.4428 \frac{b w}{R^{3}} D_{22}\left(1+f_{(w)}\right)
$$

where $f_{(w)}$ is the unknown parameter, which depends on the radial displacement $w$.

\subsection{Buckling analysis}

The manufactured cylinders were dimensioned to collapse by buckling prior to material failure, that is, the radius to thickness dimensional ratio was lower than 20 (i.e. R/t $<20$ ). The mean cylinder nominal dimensions were: $136.2 \mathrm{~mm}$ internal diameter, $200 \mathrm{~mm}$ length, and 2.6 $\mathrm{mm}$ thickness. The tube ends were closed using steel flanges with O-rings for sealing, respecting the simply-supported boundary condition. The tube was tested in a hydrostatic water chamber (Figure 2a-b) at the Subsea Technology Laboratory/UFRJ at room temperature and using a pressure increase rate of $0.4 \mathrm{MPa} / \mathrm{min}$ until the pressure dropped in the chamber.

Finite element analysis (FEA) was used to predict buckling of the composite tube when subject to an external hydrostatic pressure, using simply-supported boundary conditions on the tube edges, quadratic shell elements, and mesh size defined after conducting a mesh sensitivity analysis. For comparison purposes, FEA buckling analyses were performed using the linear bifurcation method to extract the eigenvalues of deformation modes (i.e. a linear buckling analysis) and the incremental RIKS method with geometric imperfections implementation (i.e. a non-linear buckling analysis) [8].

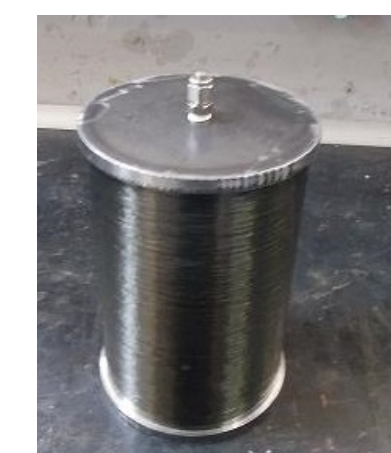

(a)

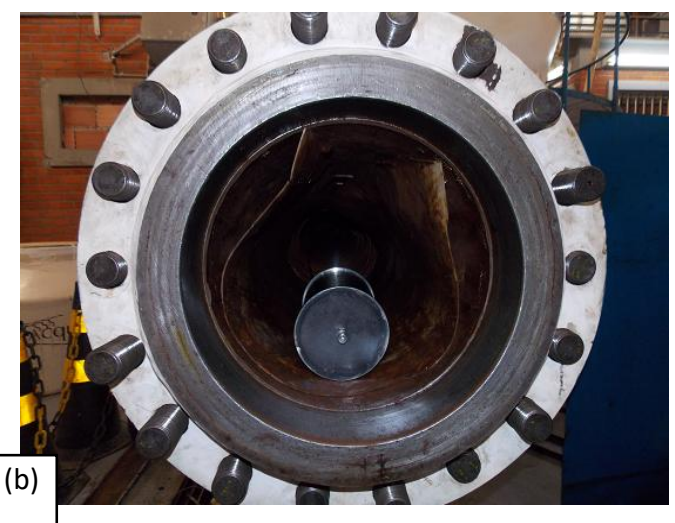

Figure 2 - Composite tube with flanges assembled (a) and placed inside the hydrostatic chamber (b).

\section{RESULTS AND DISCUSSION}

Results from the monotonic and cyclic ring compression test are shown in Figure 3. Averaged measured ring specimens dimensions were $136.2 \mathrm{~mm}$ internal diameter, $20.0 \mathrm{~mm}$ width and $2.5 \mathrm{~mm}$ thickness for the monotonic test, and $136.2 \mathrm{~mm}$ internal diameter, $21.5 \mathrm{~mm}$ width, and $2.25 \mathrm{~mm}$ thickness for the cyclic test. The differences on width and thickness of the tests specimens resulted in force-displacement curves with distinct inclinations as seen on Figure 3. The cyclic curve does not show any evidence of progressive damage until the first failure and validates 
the linear elastic behavior of the material within that range of displacement. This condition was considered in the surrogate model.

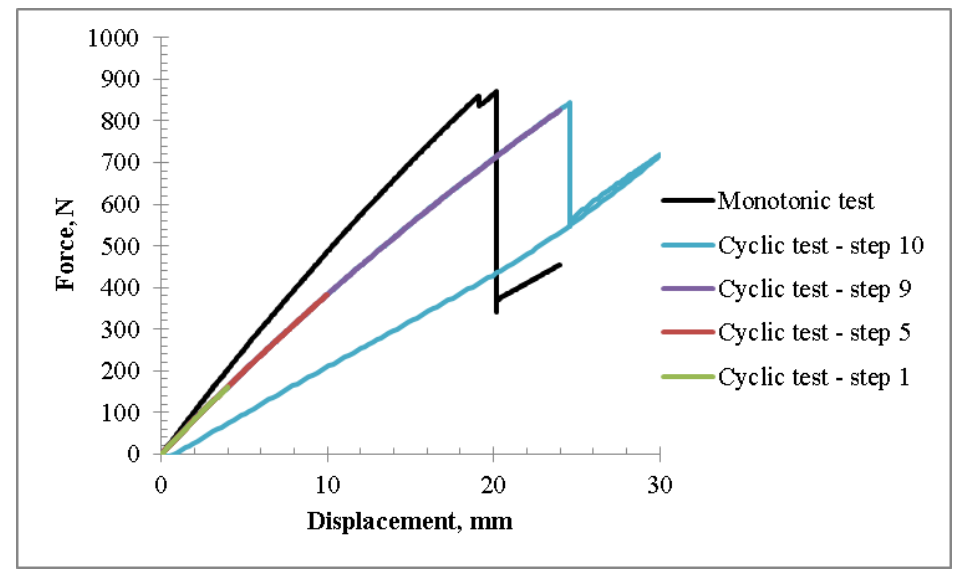

Figure 3 - Monotonic and cyclic ring compression test results.

Applying the previous characterized ply elastic properties (presented in Table 1) in the FEA simulation of the ring compression test, the force-displacement curve was obtained. The composite stiffness parameter $D_{22}$ was determined by classical laminate theory and the surrogate model developed for this study was fitted according to Equation (9). Ring dimensions used in the calculations were $136.2 \mathrm{~mm}$ internal diameter, $20.0 \mathrm{~mm}$ width and $2.5 \mathrm{~mm}$ thickness. This solution is presented in Figure 4a, which shows that the surrogate model represents well the physical behaviour. The unknown function $f_{(w)}$ is presented in Figure 4b.

Table 1: Elastic properties from flat coupons and calculated trace parameters.

\begin{tabular}{ccccccccc}
\hline $\mathrm{E}_{\mathrm{x}}$ & $\mathrm{E}_{\mathrm{y}}$ & $v_{\mathrm{xy}}$ & $\mathrm{G}_{\mathrm{xy}}$ & $\mathrm{Q}^{*}{ }_{\mathrm{xx}}$ & $\mathrm{Q}^{*}{ }_{\mathrm{yy}}$ & $\mathrm{Q}^{*}{ }_{\mathrm{xy}}$ & $\mathrm{Q}_{\text {ss }}^{*}$ & $\mathrm{Tr}$ \\
$\mathrm{MPa}$ & $\mathrm{MPa}$ & - & $\mathrm{MPa}$ & - & - & - & - & $\mathrm{MPa}$ \\
\hline 129300 & 9110 & 0.32 & 5440 & 130181 & 9172 & 2843 & 5440 & 150233 \\
\hline
\end{tabular}
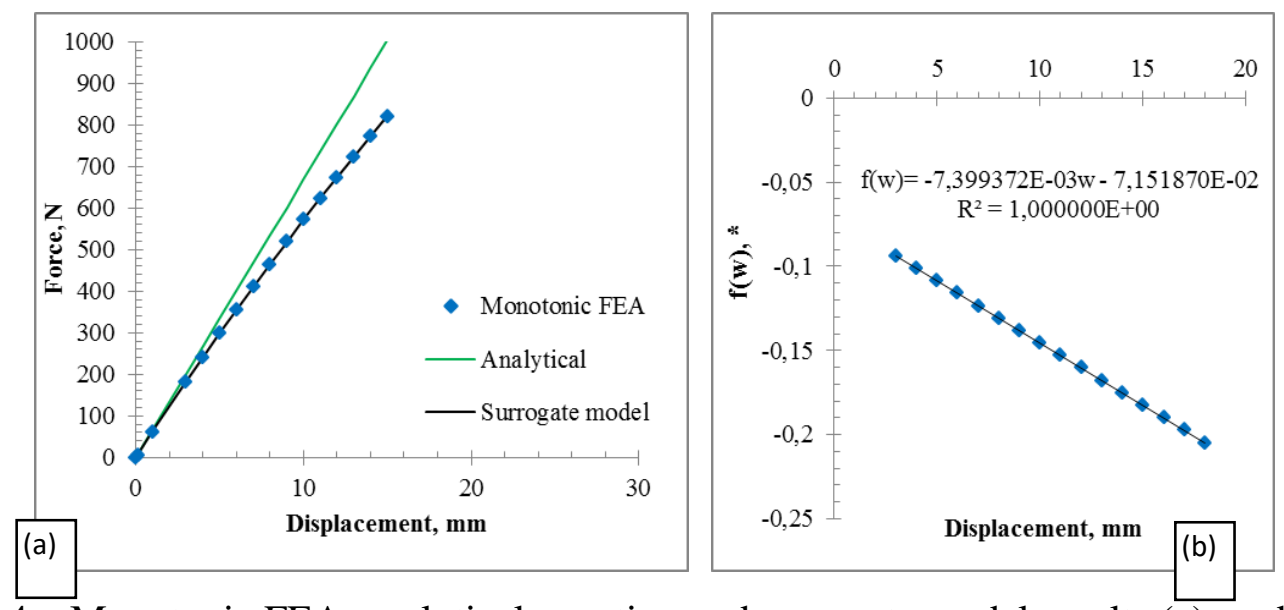

Figure 4 - Monotonic FEA, analytical equation and surrogate model results (a), and $f_{(w)}(\mathrm{b})$.

The surrogate model was applied to calculate the actual bending-stiffness parameter $D_{22}$ of the compression test results by fitting the experimental curves, as presented in Figure 5. 


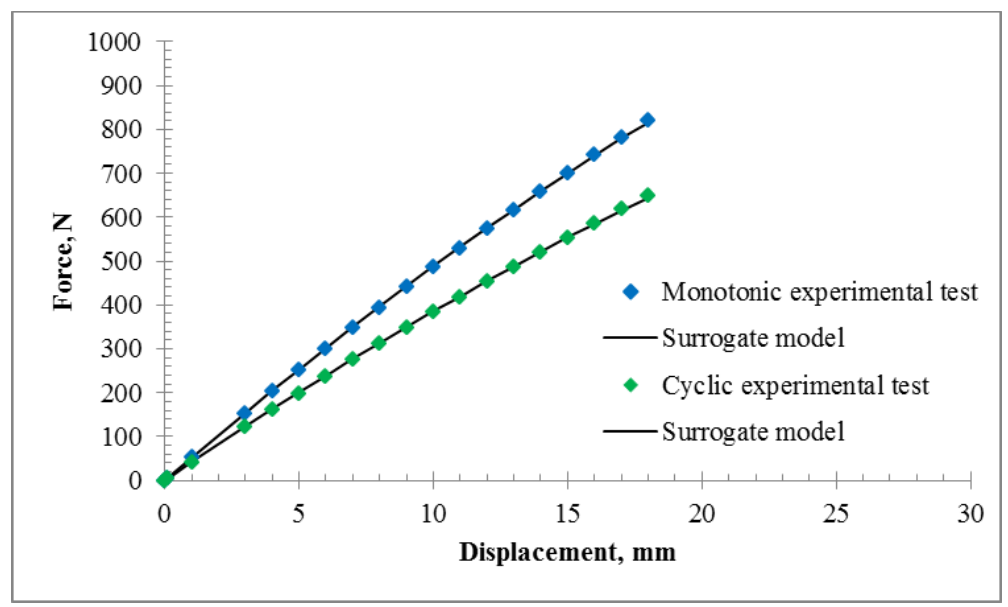

Figure 5 - Fitted surrogate model for the ring compression experimental test results.

For scaling the elastic properties, the trace-design parameters were calculated based on the estimated material properties of Table 1 to allow prediction of the as built ply material properties. These results are presented in Table 2.

Table 2: Trace flexural parameters and scaled properties of ring specimens.

\begin{tabular}{lcccccccc}
\hline & $\mathrm{D}_{22}$ & $\mathrm{D}_{22}{ }^{*}$ & $\mathrm{D}_{22}{ }^{* *}$ & $\mathrm{Tr}$ & $\mathrm{E}_{\mathrm{x}}$ & $\mathrm{E}_{\mathrm{y}}$ & $v_{\mathrm{xy}}$ & $\mathrm{G}_{\mathrm{xy}}$ \\
& $\mathrm{N} . \mathrm{mm}$ & $\mathrm{MPa}$ & - & $\mathrm{MPa}$ & $\mathrm{MPa}$ & $\mathrm{MPa}$ & - & $\mathrm{MPa}$ \\
\hline FEA & 167098 & 128331 & 0.8542 & 150233 & - & - & - & - \\
\hline Monotonic & 142583 & 109504 & 0.8542 & 128193 & 110331 & 7773 & 0.27 & 4642 \\
Cyclic & 104753 & 110357 & 0.8542 & 129192 & 111190 & 7834 & 0.27 & 4678 \\
\hline \multicolumn{8}{r}{} \\
\hline
\end{tabular}

In fact, the modulus defined with the ring compression test are an apparent modulus since due to bending we have tensile and compression regions, and a composite polymer laminate have different modulus on tensile and compression directions [10].

The mean values of the estimated elastic properties (Table 2) were used in the linear and non-linear buckling FEA model for comparison with the hydrostatic test. For the non-linear buckling FEA, percentiles of the first buckling mode shape were applied as initial imperfection in the geometry of the cylinder. Figure 6 presents the failed sample after hydrostatic test and the FEA results. Good correlation was observed between numerical and actual test results for linear as well as non-linear analysis, with $1 \%$ and $5 \%$ initial imperfections based on the first linear buckling mode.

As expected, the linear analysis resulted in a buckling load above of that of the non-linear analysis because a perfect cylindrical geometry is in general less sensitive to the load case and this is observed in the Figure 6c where higher imperfection percentiles resulted in lower buckling pressure, although in some cases an imperfection may act as reinforcement. Moreover, a 5\% imperfection based on the calculated first linear buckling mode represents here approximately an oscillation on the thickness plane of $0.125 \mathrm{~mm}$ amplitude that we can realize as a geometric deviation in the diameter consistent with manufacturing practices. Non-linear buckling result with $5 \%$ imperfection is very close to the experimental result. Geometrical imperfections must always 
be considered in a structural analysis since it is impossible to produce an exactly perfect component and moreover these imperfections affect their structural response. The ratio between experimental and linear results (knockdown-factor [10]) is 0.97 showing the accuracy of the prediction provided by the estimated material elastic properties. A linear buckling analysis with the elastic properties (Table 1) measured with the flat coupons specimens result in a buckling load of $4.88 \mathrm{MPa}$ and thus a knockdown-factor of 0.83 , almost $20 \%$ far from the experimental result against only $3 \%$ of the presented methodology.
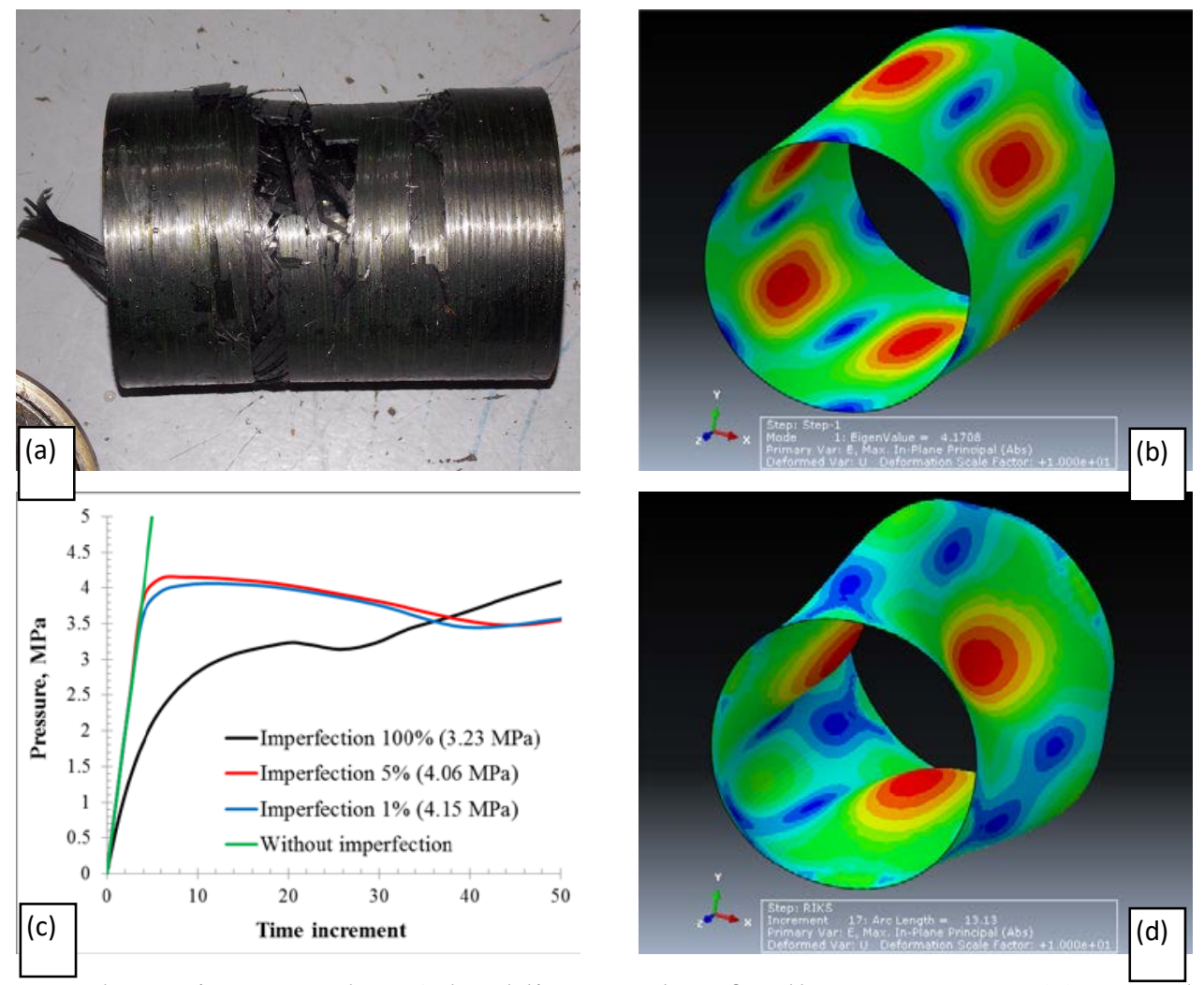

Figure 6 - Hydrostatic test and FEA buckling results of collapse pressure: (a) Tested tube (4.07 $\mathrm{MPa}$ ), (b) Linear FEA result (4.17 MPa - first mode), (c) Non-linear FEA results for imperfect geometry, (d) Non-linear buckling mode with 5\% initial imperfection (4.06 MPa).

\section{CONCLUSIONS}

The developed surrogate model has overcome the lack of accuracy of the referenced analytical model in representing the physical behaviour of the radial ring compression (parallelplate) test. Moreover, it has simplified calculations in the fitting procedure, avoiding timeconsuming FEA routine and supporting the trace-based approach. The developed scaling procedure for the elastic properties using the trace approach was validated by the ring compression test and by the experimental and FEA buckling analysis since good agreement was found in the results. This methodology was may be a valuable tool in the early stages of structural design since it minimizes the required material characterization efforts and saves time. Further investigations on the trace-design approach will enlighten its statistical basis and its correlation with composite material physical constitutive laws. 


\section{ACKNOWLEDGEMENTS}

We would like to thank the testing support of the Subsea Technology Laboratory of UFRJ, and the Composite Materials Group/LAPOL/UFRGS for cylinder manufacturing.

\section{REFERENCES}

[1] PETERS, S.T., ‘Composite Filament Winding’. ASM International, 2011.

[2] TSAI, S.W., MELO, J.D.D., ‘An invariant-based theory of composites'. Composites Science and Technology, v. 100, p. 237-243, 2014.

[3] HA, S.K., CIMINI JR, C.A., 'Theory and validation of the master ply concept for invariant-based stiffness of composites'. Journal of Composite Materials, DOI: 10.1177/0021998317728782, 2017.

[4] TSAI, S.W., SIHN, S., MELO, J.D. D., 'Trace-based stiffness for a universal design of carbon-fiber reinforced composite structures’. Composites Science and Technology, v. 118, p. 23-30, 2015.

[5] CHOQUEUSE, D., DAVIES, P. ‘Durability of Composite Materials for Underwater Applications’. In: Durability of Composites in a Marine Environment. Springer, Dordrecht, 2014. p. 195-207.

[6] MELO, J.D., BI, J., TSAI, S.W., 'A novel invariant-based design approach to carbon fiber reinforced laminates’. Composite Structures, v. 159, p. 44-52, 2017.

[7] ALMEIDA JR, J.H.S., RIBEIRO, M.L., TITA, V., AMICO, S.C., 'Damage and failure in carbon/epoxy filament wound composite tubes under external pressure: Experimental and numerical approaches'. Materials \& Design, v. 96, p. 431-438, 2016.

[8] SYSTEMES, Dassault. ABAQUS 6.7-1 FEA software, ABAQUS. 2007.

[9] TIMOSHENKO, S.P., ‘Theory of Elastic Stability’, McGraw-Hill Book Company, 1936.

[10]GERHARDT, E., AMICO, S., MARCZAK, R., 'Numerical and computational analysis regarding buckling knockdown factor of composite cylinders'. In: MACMS - Meeting on Aeronautical Composite Materials and Structures, Brazil, 2017. Available at: http://soac.eesc.usp.br/index.php/macms/macms2017/paper/view/820. Accessed: 19 Feb. 2018. 